\title{
Connections on Discrete Fibre Bundles
}

\author{
N. S. Manton \\ St. John's College, Cambridge CB2 1TP, United Kingdom, and \\ Department of Applied Mathematics and Theoretical Physics, Silver Street, \\ Cambridge CB3 9EW, United Kingdom
}

\begin{abstract}
A new approach to gauge fields on a discrete space-time is proposed, in which the fundamental object is a discrete version of a principal fibre bundle. If the bundle is twisted, the gauge fields are topologically non-trivial automatically.
\end{abstract}

\section{Introduction}

For both computational and conceptual purposes it is very often convenient to formulate physical theories on a space or space-time lattice. A lattice discretizes space but preserves some of its topological structure through the provision of links between neighbours of the lattice. A smooth curve is approximated not by an arbitrary set of lattice points but by points joined by these links and this allows one to determine, for example, whether a closed loop in a 3-dimensional lattice is knotted or not. By a lattice, one usually means an infinite set of points in $\mathbb{R}^{n}$ generated by a discrete translation group. We shall actually be interested in compact, spherical spaces and shall use the word lattice to mean a finite set of points on the sphere generated by a discrete subgroup of the rotation group.

In this paper, we propose a new way to consider lattice gauge fields. Existing treatments are not really satisfactory because the topological properties of continuum gauge fields are easily lost in the discretization of space. This leads to difficulties with fermions on the lattice; it seems impossible to incorporate chiral fermions, and the anomalies of the continuum theory disappear.

Lüscher [1] has proposed a definition of the topological charge of a lattice gauge field in four dimensions, which has been developed and implemented numerically by Woit [2], Phillips and Stone [3], Teper [4] and others. A characteristic feature of Lüscher's idea is that, locally, in a suitable gauge, the group elements on the links of the lattice (Wilson link variables) which define the lattice gauge field are close to the identity. However, there are difficulties. The definition fails for some exceptional configurations, and it is algebraically rather complicated. Also, the underlying fibre bundle is not fundamental in Lüscher's 
scheme, being instead pieced together from locally trivial bundles. Nevertheless, we shall see that our proposal has something in common with Lüscher's.

It is well known by now that topologically non-trivial gauge fields are best discussed as connections on a principal fibre bundle [5]. The base space represents space or space-time and each fibre is a copy of the gauge group. The bundle picture is not just more natural, but it accommodates symmetries more easily. The bundle can have a bigger symmetry group than simply the product of the gauge group and the symmetry group of the base space.

Our new approach to lattice gauge fields consists in taking a discrete total space for the bundle, having a finite number of points, The gauge group is a discrete group acting on these points. The fibres are the orbits of this group, and the base space is the quotient of the bundle by the group action. There is therefore one point in the base space for each fibre in the bundle. The bundle need not have a simple product structure. Discrete gauge groups have appeared before in lattice gauge theory, especially in computational work, but only in the context of a trivial bundle.

We suppose that the bundle comes equipped with links connecting neighbouring points, and that under the action of the gauge group, links are mapped into links. We shall define which points in the bundle are neighbours by making use of a standard Riemannian metric on the space which the discrete bundle is approximating. Two fibres are defined to be adjacent, and the corresponding base points are linked, if and only if some pair of points in these fibres are neighbours in the bundle. The base space is therefore a lattice in the usual way, but its link structure is determined by that of the bundle.

In a smooth bundle, a connection is a rule for lifting an infinitesimal step in the base space to an infinitesimal step in the bundle between points on the corresponding fibres. On a discrete bundle a connection is defined analogously to be a rule for lifting a link in the base to a link in the bundle.

We shall concentrate in this paper on two particularly nice examples of discrete bundles, which arise by making a discretization of the familiar smooth Hopf bundles $[5,6]$ :

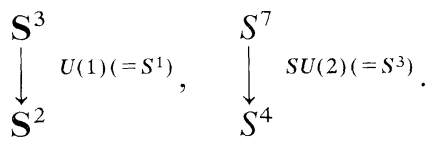

Both are topologically non-trivial, i.e. $S^{3} \neq S^{2} \times S^{1}$ and $S^{7} \neq S^{4} \times S^{3}$. The first has a connection which is equivalent to the magnetic field through a 2 -sphere which encloses a Dirac monopole; the second has a connection which is equivalent to an instanton. These bundles are therefore familiar to physicists.

A fundamental consequence of the fact that the Hopf bundles are twisted is that there is no flat connection on them. Our discrete versions of these bundles will have the same property. The holonomies (Wilson loops) around elementary triangles in the base space cannot all be unity.

A more profound relationship between connections and the' topology of smooth bundles is through the Chern numbers [5]. These integers are the integrals of the Chern forms, which are closed differential forms of even degree on the base space, constructed from the curvature 2-form $F$. Each Chern number is the same for all connections and depends only on the topology of the bundle and its 
fibration. If any Chern number is non-zero, the bundle is twisted. On the $U(1)$ Hopf bundle, the first Chern number is 1 . It is the integral of the 2-form $\frac{1}{2 \pi} F$ over $S^{2}$ and is physically identified with twice the magnetic charge inside the sphere. On the $S U(2)$ Hopf bundle the second Chern number is 1. It is the integral of the 4-form $\frac{1}{8 \pi^{2}} \operatorname{Tr}(F \wedge F)$ over $S^{4}$, and is identified with the instanton number.

On the discrete version of the $U(1)$ Hopf bundle, we have found the analogue of the first Chern form. Its integral is 1 for all connections. On the discrete version of the $S U(2)$ bundle, however, we have not found an appropriate analogue of the second Chern form, although one may exist. Despite this difficulty, we stress that our bundle is certainly twisted, and believe that with sufficient ingenuity, the analogue of $\operatorname{Tr}(F \wedge F)$ can be found.

Section 2 is a review of the smooth Hopf bundles. In Sect. 3 we describe a discrete version of the $U(1)$ Hopf bundle with its connections, and in Sect. 4 a discrete version of the $S U(2)$ Hopf bundle with its connections. We hope that the methods introduced here can be extended to a much larger class of discrete fibre bundles, so as to be useful in the numerical studies of quantized lattice gauge theories. We also think it will be interesting to explore the implications of discrete twisted bundles for fermions interacting with gauge fields.

\section{The Hopf Bundles [6, Sect. 20]}

The total space of the $U(1)$ Hopf bundle is the unit 3 -sphere $S^{3}$. This is identified with the set of ordered pairs of complex numbers $\left(z_{1}, z_{2}\right)$ subject to the constraint

$$
z_{1} \bar{z}_{1}+z_{2} \bar{z}_{2}=1 \text {. }
$$

Multiplication by complex numbers of unit magnitude, according to the rule

$$
\left(z_{1}, z_{2}\right) \stackrel{e^{i \lambda}}{\longrightarrow}\left(z_{1} e^{i \lambda}, z_{2} e^{i \lambda}\right)
$$

preserves this constraint, and gives an action of $U(1)$ on $S^{3}$. There are no fixed points if $e^{i \lambda} \neq 1$, so $S^{3}$ is fibred as a bundle of circles. The complex number $Z=z_{1} z_{2}^{-1}$ is constant on each fibre and specifies the fibre uniquely. $Z$ is assigned the value $\infty$ on the fibre consisting of the points $\left(e^{i \alpha}, 0\right)$. $Z$ therefore takes any value in the extended complex plane, which is topologically $S^{2}$, the Riemann sphere.

The Hopf bundle is twisted and has no global section. On any circle $|Z|=R(0<R<\infty)$, traversed once anti-clockwise, $\arg Z$ increases by $2 \pi$. Therefore, on a smooth section $Z \rightarrow\left(z_{1}(Z), z_{2}(Z)\right)$, $\arg z_{1}$ increases by $2 \pi(n+1)$ and $\arg z_{2}$ increases by $2 \pi n$, for some integer $n$. If the section can be extended to the fibre $Z=0$, it will pass through a point $\left(0, e^{i \alpha}\right)$ for some $\alpha$. By continuity, $z_{2}$ remains close to $e^{i \alpha}$ on circles with $R$ small, so $n$ must be zero. A similar argument shows that if the section can be extended to the fibre $Z=\infty$, then $n+1$ is zero. $n$ cannot be both 0 and -1 , so no global sections exist. Two local sections, which together cover the whole base space, and which overlap on the equator of the Riemann sphere, $|Z|=1$, certainly can be found. The transition between them defines a map from the 
equator to the group $U(1)$, i.e. a map from a circle to a circle. This map has winding number 1.

The $S U(2)$ Hopf bundle has a very similar structure with complex numbers replaced by quaternions. The total space is now the 7 -sphere $S^{7}$, realized as ordered pairs of quaternions $\left(q_{1}, q_{2}\right)$ subject to the constraint

$$
q_{1} \bar{q}_{1}+q_{2} \bar{q}_{2}=1 \text {. }
$$

The unit quaternions $\{q: q \bar{q}=1\}$ form the group $S U(2)$. They act by right multiplication on pairs of quaternions

$$
\left(q_{1}, q_{2}\right) \stackrel{q}{\longrightarrow}\left(q_{1} q, q_{2} q\right)
$$

preserving the constraint (2.3), and again there are no fixed points if $q \neq 1 . S^{7}$ is therefore a principal $S U(2)$ bundle. The quaternion $Q=q_{1} q_{2}^{-1}$ is invariant on each fibre, since

$$
\left(q_{1} q\right)\left(q_{2} q\right)^{-1}=q_{1} q_{2}^{-1}
$$

and $Q$ uniquely specifies the fibre. $Q$ can be any quaternion including $\infty$. Since the one-point compactification of $\mathbb{R}^{4}$ is $S^{4}$, the base space of the bundle is $S^{4}$. The base may be covered by two local sections which overlap on the equatorial $S^{3}$, i.e. where $|Q|=1$. The transition between them is a map from $S^{3}$ to $S U(2)$, i.e. a map from $S^{3}$ to $S^{3}$. This map has degree 1 .

\section{Connections on a Discrete $U(1)$ Hopf Bundle}

We take a 24-point discretization of $S^{3}$ as the total space of the bundle. The points have Cartesian coordinates

$$
\begin{array}{ll}
( \pm 1,0,0,0), & (0, \pm 1,0,0), \quad(0,0, \pm 1,0), \\
(0,0,0, \pm 1), & \frac{1}{2}( \pm 1, \pm 1, \pm 1, \pm 1),
\end{array}
$$

and are the vertices of a regular self-dual polytope in $\mathbb{R}^{4}[7]$. Presented as pairs of complex numbers $\left(z_{1}, z_{2}\right)$, these points are

$$
( \pm 1,0), \quad( \pm i, 0), \quad(0, \pm 1), \quad(0, \pm i), \quad \frac{1}{2}( \pm 1 \pm i, \pm 1 \pm i) .
$$

The gauge group is the $\mathbb{Z}_{4}$ subgroup of $U(1)$, which acts as in (2.2),

$$
\left(z_{1}, z_{2}\right) \stackrel{g}{\longrightarrow}\left(z_{1} g, z_{2} g\right), \quad g \in\{1, i,-1,-i\} .
$$

There are six orbits of four points each, which are the fibres of the bundle. The base space therefore has six points. The fibre invariant $Z=z_{1} z_{2}^{-1}$ takes the values $\{\infty, \pm 1, \pm i, 0\}$. We use $Z$ to denote both the fibre and the corresponding base point, and as a subscript to denote on which fibre a point in the bundle lies. As points on the Riemann sphere the six base points are the vertices of an octahedron, the opposing pairs of vertices being $(1,-1),(i,-i)$ and $(\infty, 0)$. 
Each point in the bundle has eight nearest neighbours at unit separation. The neighbours of $(1,0)_{\infty}$, for example, are

$$
\begin{array}{llll}
\frac{1}{2}(1+i, 1+i)_{1}, & \frac{1}{2}(1+i, 1-i)_{i}, \quad \frac{1}{2}(1+i,-1-i)_{-1}, \quad \frac{1}{2}(1+i,-1+i)_{-i}, \\
\frac{1}{2}(1-i, 1-i)_{1}, & \frac{1}{2}(1-i,-1-i)_{i}, \quad \frac{1}{2}(1-i,-1+i)_{-1}, \quad \frac{1}{2}(1-i, 1+i)_{-i} .
\end{array}
$$

There are two neighbours on each of the four fibres $1, i,-1,-i$, but none on the fibre 0 . The pair of neighbours on the same fibre are always related by multiplication by $\pm i$. In general, a point has no neighbours on its own fibre or on the one opposite, but two neighbours on each of the remaining fibres. This establishes that the links in the base space are all the usual edges of the octahedron.

Let us now define what we mean by a section of this discrete bundle. It is the analogue of a smooth section in the usual Hopf bundle. A set of points in the bundle, with no more than one from each fibre, is called a section if it is a set of mutual neighbours, that is, if each pair of points on adjacent fibres are neighbours in the bundle. A global section of six points does not exist, but one may find local sections which omit one fibre. For example, there is a local section omitting the fibre 0 :

$$
\begin{aligned}
& (1,0)_{\infty}, \quad \frac{1}{2}(1+i, 1+i)_{1}, \quad \frac{1}{2}(1+i, 1-i)_{i}, \quad \frac{1}{2}(1+i,-1-i)_{-1}, \\
& \frac{1}{2}(1+i,-1+i)_{-i},
\end{aligned}
$$

but no way of extending this to a global section. The absence of a global section may be verified as follows. Suppose a section contains the points $(a, 0)_{\infty}$ and $(0, b)_{0}$, where $a$ and $b$ are in the set $\{1, i,-1,-i\}$. The four points on the equatorial fibres which are neighbours of both $(a, 0)_{\infty}$ and $(0, b)_{0}$ are $\frac{1}{2}(a \pm i a, b \pm i b)$. Two of these points lie on the fibre $a b^{-1}$, one on $i a b^{-1}$, one on $-i a b^{-1}$, but none on $-a b^{-1}$. The section cannot therefore be extended to all fibres.

A local section on the fibres $1, i,-1,-i$, and 0 is

$$
\begin{array}{ll}
\frac{1}{2}(1+i, 1+i)_{1}, & \frac{1}{2}(-1+i, 1+i)_{i}, \quad \frac{1}{2}(-1-i, 1+i)_{-1}, \\
\frac{1}{2}(1-i, 1+i)_{-i}, & (0,1)_{0} .
\end{array}
$$

The sections (3.5) and (3.6) overlap on the equatorial fibres where they are related by transition elements of the gauge group as follows:

$$
\begin{gathered}
\frac{1}{2}(1+i, 1+i)_{1} \stackrel{1}{\longrightarrow} \frac{1}{2}(1+i, 1+i)_{1}, \\
\frac{1}{2}(1+i, 1-i)_{i} \stackrel{i}{\longrightarrow} \frac{1}{2}(-1+i, 1+i)_{i} . \\
\frac{1}{2}(1+i,-1-i)_{-1} \stackrel{-1}{\longrightarrow} \frac{1}{2}(-1-i, 1+i)_{-1}, \\
\frac{1}{2}(1+i,-1+i)_{-i} \stackrel{-i}{\longrightarrow} \frac{1}{2}(1-i, 1+i)_{-i} .
\end{gathered}
$$

The map from the four-point equator to the gauge group is the identity map.

In the usual Hopf bundle the corresponding map from $S^{1}$ to $S^{1}$ has winding number 1 and is the identity map for suitably chosen local sections. A map from a four-point discretization of the circle to itself has a well-defined winding number 1 , 
0 or -1 , provided that the images of two adjacent points are always either identical or adjacent. Overlapping local sections of our discrete bundle give rise to a map of this sort. This is because the local sections both consist of mutual neighbours, so the transition elements of the gauge group can only differ by $1, i$ or $-i$, and not by -1 , on adjacent fibres. The discrete identity map has winding number 1 , and this is a measure of the non-triviality of our discrete bundle.

We now define a connection. A connection is an equivariant rule for lifting links between neighbours in the base space to links in the bundle. Consider neighbours in the base space $Z$ and $\tilde{Z}$, and the fibres over them. A point $p$ in the fibre $Z$ has two neighbours $\tilde{p}_{1}$ and $\tilde{p}_{2}$ in the fibre $\tilde{Z}$. The step from $Z$ to $\tilde{Z}$ in the base may be lifted to a step from $p$ to $\tilde{p}_{1}$ or from $p$ to $\tilde{p}_{2}$. The connection specifies which step is to be made. The starting point on the fibre $Z$ need not be $p$; it could be a different point $p g$, where $g$ is an element of the gauge group. Equivariance means that if the connection specified a step from $p$ to $\tilde{p}_{1}$ then the step from $p g$ to $\tilde{p}_{1} g$, and similarly for the other possibility. The connection is thus invariant under the action of the gauge group on the bundle.

As an example, consider the connections on the link $\infty \rightarrow 1$ in the base. The eight points on the fibres $\infty$ and 1 are shown below. One connection is indicated by the dashed lines, the other by the dotted lines.

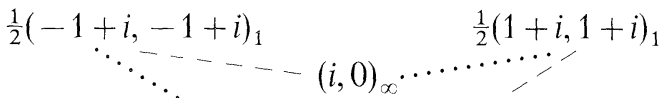

$$
\begin{aligned}
& (-1,0)_{\infty} \quad(1, \overline{0})_{\infty}
\end{aligned}
$$

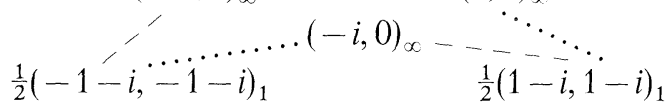

The connection on the step from $\tilde{Z}$ to $Z$ simply reverses the effect of the step from $Z$ to $\tilde{Z}$. Thus a step in the base from $Z$ to $\tilde{Z}$ and back is lifted to a step from $p$ to $\tilde{p}_{1}$ and back, or from $p$ to $\tilde{p}_{2}$ and back. There are 12 links in the base space of our bundle, since an octahedron has 12 edges. On each there are two choices for the connection, so there are $2^{12}$ connections altogether.

A continuous path of several links in the base may be lifted to the bundle using a connection. One must again specify the starting point of the lift of the first link, but each subsequent link in the bundle starts where the previous one ended. In this way one gets a continuous path in the bundle. A closed loop in the base is lifted to a path in the bundle whose starting point and finishing point are on the same fibre, but the lifted path need not be closed. The finishing point is related to the starting point by an element of the gauge group which is called the holonomy on the loop. Because the gauge group is abelian, and because the connection is equivariant, the holonomy depends only on the loop. It doesn't depend on where we say it starts and finishes, nor on the point in the bundle to which the starting point is lifted.

Holonomy measures the curvature of the connection; the connection is flat if the holonomy on all closed loops is the identity element of the gauge group. If a flat connection is used to lift all possible paths starting at $Z$ in the base to the paths starting at $p$ (on the fibre $Z$ ) in the bundle, then a global section of the bundle 
through $p$ is generated. Conversely, a global section defines a flat connection. Since our bundle has no global section, it cannot in fact have any flat connection.

Let us look at the possible holonomies on an elementary triangle in the base, for example, the one with vertices $\infty, 1$, and $i$. There are eight different connections on this triangle. Suppose we lift the path $\infty \rightarrow 1 \rightarrow i \rightarrow \infty$ to the bundle, starting at $(1,0)_{\infty}$. The possible paths in the bundle can be determined from the diagram below:

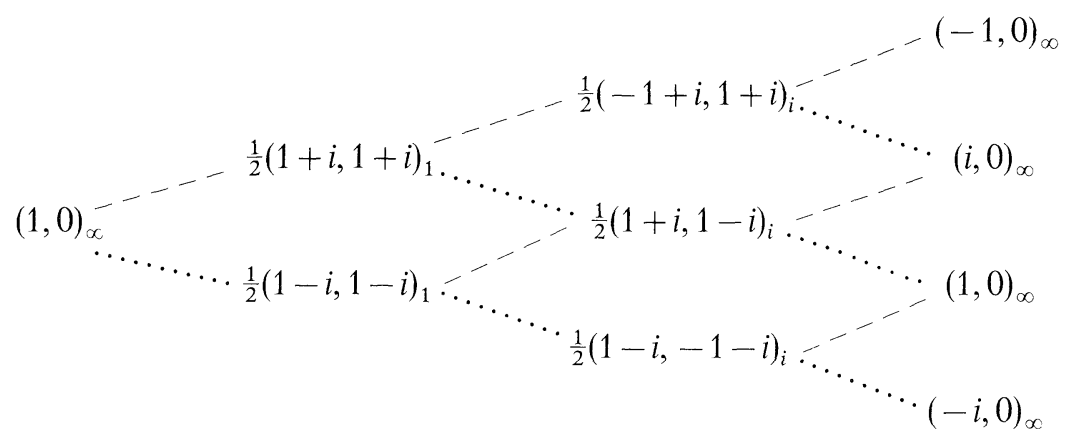

Three of the connections lead to $(1,0)_{\infty}$ and produce a closed loop in the bundle, three lead to $(i, 0)_{\infty}$, one to $(-1,0)_{\infty}$ and one to $(-i, 0)_{\infty}$. The holonomy is 1 or $i$ in three ways, and -1 or $-i$ in one way. There is clearly an asymmetry. We can unambiguously define the phases of the holonomies $-i, 1, i$, and -1 to be $-\frac{1}{2} \pi, 0$, $\frac{1}{2} \pi$, and $\pi$, respectively. (Choosing the phase of -1 to be $-\pi$ is not reasonable, as the connection giving holonomy -1 differs from the connections giving holonomy $i$ on only one link, but differs from the connection giving holonomy $-i$ on all three links.) This phase may be identified with the magnetic flux through the triangle. The average magnetic flux for all eight connections is $\frac{1}{4} \pi$. This is not a special property of the triangle considered; the average flux is the same through any triangle oriented the same way, and is a consequence of the twist of the bundle.

The octahedron has eight triangular faces, and the average magnetic flux, summed over these faces, is $2 \pi$. But more is true. For any particular connection, the total flux is $2 \pi$. This is the discrete analogue of the result that the smooth Hopf bundle's first Chern number is 1 . To verify that

$$
\frac{1}{2 \pi} \sum_{\text {faces }} \text { magnetic flux }=1
$$

on the discrete bundle, one needs to first check the result for one particular connection. This is easily done. Any other connection can be obtained from this one by making changes on one link at a time. But a change on one link only changes the holonomy on the two triangles whose boundary contains that link. On one triangle the flux increases by $\frac{1}{2} \pi$, and on the other, where the link has the opposite orientation, it decreases by $\frac{1}{2} \pi$. The sum of the fluxes on all triangles is unaltered. Equation (3.10) is therefore true for all connections.

Our description of a connection can be related to Wilson's description of a lattice gauge field, where an element of the gauge group is associated to each link in the base space and holonomies are given by products of these group elements [8], 
by using local sections. This is completely analogous to the usual situation in a smooth fibre bundle. There, the connection becomes a conventional gauge potential only relative to a smooth local section of the bundle, and on a twisted bundle the gauge potential cannot be globally defined.

Suppose we are given a local section which contains points $p$ and $\tilde{p}$ on adjacent fibres $Z$ and $\tilde{Z}$. There are two points on the fibre $\tilde{Z}$ which are neighbours of $p$ in the bundle. One is $\tilde{p}$, since a section consists of mutual neighbours; the other is either $\tilde{p} i$ or $\tilde{p}(-i)$ depending on the choice of section. If the connection specifies that the link $Z \rightarrow \widetilde{Z}$ is lifted to the link $p \rightarrow \tilde{p}$, then it is represented on the link $Z \rightarrow \widetilde{Z}$ by the group element 1 . If the connection specifies a lift to the link $p \rightarrow \tilde{p} i[$ or $p \rightarrow \tilde{p}(-i)]$, then it is represented by $i[$ or $-i]$. As in Lüscher's prescription for lattice gauge fields, these group elements are near 1 , in that -1 never occurs. However, there is an asymmetry here arising from the twist of the bundle; only one of the two group elements $i$ or $-i$ is allowed.

Transforming from one local section to another on the same fibres may be regarded as a gauge transformation, but unlike in conventional lattice gauge theory, gauge transformations using arbitrary elements of the gauge group are not permitted. The gauge transformation must take neighbours to neighbours in the bundle. Otherwise the residual continuity that exists on the discrete bundle would be destroyed.

To describe the connection completely in terms of group elements on the links, we need not to use two local sections which cover the base space, for example, the sections (3.5) and (3.6). On a link covered by both sections, the group elements relative to the two sections differ, and are related by the transition elements given in (3.7). If the group element on the link $Z \rightarrow \tilde{Z}$ relative to the first section is $g_{1}$, and relative to the second section is $g_{2}$, and if the transition elements from the first to the second section are $g(Z)$ on fibre $Z$ and $g(\tilde{Z})$ on fibre $\widetilde{Z}$, then

$$
g_{2}=g(\tilde{Z})^{-1} g_{1} g(Z) \text {. }
$$

As an example of a connection, suppose that relative to the section (3.5) on the hemisphere containing $\infty$, all group elements are 1 . The holonomies on the four triangles meeting at $\infty$ are all 1 , and there is no magnetic flux. Relative to section (3.6) on the hemisphere containing 0, suppose that the group elements are 1 on all links which end at 0 ; on the remaining equatorial links, which are common to both hemispheres, the group elements are determined by formula (3.11) to be $i$. The holonomy is therefore $i$ on all four triangles in the hemisphere containing 0 , and the magnetic fluxes are $\frac{1}{2} \pi$. The total magnetic flux is $2 \pi$.

\section{Connections on a Discrete $S U(2)$ Hopf Bundle}

This is in many ways similar to the discrete $U(1)$ Hopf bundle but with complex numbers replaced by quaternions. In the usual $S U(2)$ Hopf bundle the total space is the 7-sphere, the base space is the 4-sphere, and the gauge group is $S U(2)$. As a discrete approximation to $S^{7}$ we shall take the 240 points which are the vertices of Gosset's semi-regular polytope in $\mathbb{R}^{8}[7,9]$. These points are nowadays more familiar as the roots of $E_{8}$. The discrete gauge group is a 24-element non-abelian 
subgroup of $S U(2)$. As a manifold, $S U(2)$ is the 3-sphere, and the 24 points in the subgroup are precisely the points on $S^{3}$ that formed the total space of the discrete $U(1)$ Hopf bundle. This group acts on the 240 points on $S^{7}$ through quaternion multiplication, and there are 10 orbits. The base space of our bundle is therefore a 10-point discretization of $S^{4}$.

We present the 240 points on $S^{7}$ relative to Cartesian unit vectors $\left\{e_{i}: i=1, \ldots, 8\right\}$ first. They are

(a) $\pm e_{1}, \pm e_{2}, \pm e_{3}, \pm e_{4}, \frac{1}{2}\left( \pm e_{1} \pm e_{2} \pm e_{3} \pm e_{4}\right)$,

(b) $\pm e_{5}, \pm e_{6}, \pm e_{7}, \pm e_{8}, \frac{1}{2}\left( \pm e_{5} \pm e_{6} \pm e_{7} \pm e_{8}\right)$,

(c) $\frac{1}{2}\left( \pm e_{a} \pm e_{b} \pm e_{c} \pm e_{d}\right)$,

where either

$$
\left(c_{1}\right)(a, b)=(1,2) \quad \text { or }(3,4), \quad \text { and }(c, d)=(5,6) \text { or }(7,8)
$$

or

$$
\left(\mathrm{c}_{2}\right)(a, b)=(1,3) \quad \text { or }(2,4), \quad \text { and }(c, d)=(5,7) \text { or }(6,8)
$$

or

$$
\left(c_{3}\right)(a, b)=(1,4) \text { or }(2,3) \text {, and }(c, d)=(5,8) \text { or }(6,7) .
$$

There are 24 points of type (a) and 24 of type (b) and 64 each of types $\left(\mathrm{c}_{1}\right),\left(\mathrm{c}_{2}\right)$, and $\left(c_{3}\right)$. This is how the roots of $E_{8}$ split relative to the subgroup $\operatorname{Spin}(8) \times \operatorname{Spin}(8) / \mathbb{Z}_{2}$ $\times \mathbb{Z}_{2}$.

A point $\sum_{1}^{8} \alpha_{m} e_{m}$ in $\mathbb{R}^{8}$ can be identified with a pair of quaternions $\left(q_{1}, q_{2}\right)$, where $q_{1}=\alpha_{1}+\alpha_{2} i+\alpha_{3} j+\alpha_{4} k$ and $q_{2}=\alpha_{5}+\alpha_{6} i+\alpha_{7} j+\alpha_{8} k$. If the point is on the unit 7 -sphere, then $q_{1} \bar{q}_{1}+q_{2} \bar{q}_{2}=1$. From now on we identify the 240 points (4.1) with pairs of quaternions in this way. Right multiplication by a unit quaternion permutes the 240 points among themselves if it belongs to the 24-element subgroup of $S U(2)$,

$$
H=\left\{ \pm 1, \pm i, \pm j, \pm k, \frac{1}{2}( \pm 1 \pm i \pm j \pm k)\right\} .
$$

$H$ is the Hurwitz group of unit "integer" quaternions [10]. $H$ acts without fixed points, so there are ten orbits of 24 points. Each orbit is a fibre of our bundle. One fibre consists of the points of type (a), another consists of the points of type (b). The remaining eight fibres each contain eight points of type $\left(c_{1}\right)$, eight of type $\left(c_{2}\right)$, and eight of type $\left(\mathrm{c}_{3}\right)$.

The fibre invariant $Q=q_{1} q_{2}^{-1}$ has one of the ten values $\{\infty, \pm 1, \pm i, \pm j, \pm k, 0\}$, which can be thought of as points on $S^{4}$. These latter points form the base space of our bundle. They are the vertices of a polytope in $\mathbb{R}^{5}$ analogous to an octahedron in $\mathbb{R}^{3}$. Each point is adjacent to eight others, only the point opposite not being adjacent. The opposite pairs are $(\infty, 0),(1,-1),(i,-i),(j,-j)$, and $(k,-k)$. We shall refer to the fibres $\{ \pm 1, \pm i, \pm j, \pm k\}$ as the equatorial fibres. 
Examples of points on each of the ten fibres are:

$$
\begin{array}{cc}
(1,0)_{\infty}, & (0,1)_{0}, \\
\frac{1}{2}(1+i, 1+i)_{1}, & \frac{1}{2}(-1-i, 1+i)_{-1}, \\
\frac{1}{2}(-1+i, 1+i)_{i}, & \frac{1}{2}(1-i, 1+i)_{-i}, \\
\frac{1}{2}(j-k, 1+i)_{j}, & \frac{1}{2}(-j+k, 1+i)_{-j}, \\
\frac{1}{2}(j+k, 1+i)_{k}, & \frac{1}{2}(-j-k, 1+i)_{-k},
\end{array}
$$

where the final subscript indicates the fibre invariant $Q$.

Every point in the bundle is linked to 56 nearest neighbours. For example, the nearest neighbours of $(1,0)_{\infty}$ are

$$
\begin{array}{cc}
\frac{1}{2}(1 \pm i \pm j \pm k, 0), \\
\frac{1}{2}(1 \pm i, \pm 1 \pm i), & \frac{1}{2}(1 \pm i, \pm j \pm k), \\
\frac{1}{2}(1 \pm j, \pm 1 \pm j), & \frac{1}{2}(1 \pm j, \pm i \pm k), \\
\frac{1}{2}(1 \pm k, \pm 1 \pm k), & \frac{1}{2}(1 \pm k, \pm i \pm j) .
\end{array}
$$

The first eight of these points are on the fibre $\infty$; the rest are on the equatorial fibres, with six points on each. None are on the fibre 0 . This is the general pattern. Each point has eight neighbours on the same fibre, none on the opposite one, and six on each of the eight adjoining fibres. All pairs of points in the base space are therefore linked, except opposite pairs.

A connection is defined, as before, as an equivariant rule for lifting steps between neighbours in the base to steps between neighbours in the bundle. There are 40 links connecting neighbours in the base, and six choices for the connection on a link, so there are $6^{40}$ connections altogether. The existence of a flat connection would, as before, allow one to construct a global section of mutual neighbours.

We show next that there is no global section of the bundle, and hence no flat connection. Without loss of generality we can consider sections that pass through $(0,1)_{0}$, because the gauge group relates sections through other points on the fibre 0 to these. Consider a section that also passes through a point on the fibre $\infty$. There are two cases. The section may pass through $(q, 0)_{\infty}$, where $q= \pm 1, \pm i, \pm j$ or $\pm k$. The common neighbours of $(q, 0)_{\infty}$ and $(0,1)_{0}$ are the 12 points $\frac{1}{2}(q(1 \pm r), 1+r)$, where $r= \pm i, \pm j$ or $\pm k$, and they are on the fibres $Q=q(1 \pm r)(1+r)^{-1}$. There are therefore six common neighbours on the fibre $q$, one each on the fibres $\pm q i, \pm q j$, and $\pm q k$, but none on the fibre $-q$. The section cannot be extended to all ten fibres. On the other hand, the section may pass through $\frac{1}{2}\left(\alpha_{1}+\alpha_{2} i+\alpha_{3} j+\alpha_{4} k, 0\right)_{\infty}$, where $\alpha_{1}, \alpha_{2}, \alpha_{3}$, and $\alpha_{4}$ are 1 or -1 . One can verify that this point and $(0,1)_{0}$ also have 12 common neighbours on the equatorial fibres, but now there are three on each of the fibres $\alpha_{1}, \alpha_{2} i, \alpha_{3} j$, and $\alpha_{4} k$. For example, the three common neighbours on the fibre $\alpha_{1}$ are

$$
\frac{1}{2}\left(\alpha_{1}+\alpha_{2} i, 1+\alpha_{1} \alpha_{2} i\right), \quad \frac{1}{2}\left(\alpha_{1}+\alpha_{3} j, 1+\alpha_{1} \alpha_{3} j\right), \quad \frac{1}{2}\left(\alpha_{1}+\alpha_{4} k, 1+\alpha_{1} \alpha_{4} k\right) .
$$

Again the section cannot be extended to all fibres. There is therefore no global section. 
An example of a local section which excludes only the fibre 0 is the set of nine points

$$
(1,0), \quad \frac{1}{2}(1+i, \pm 1 \pm i), \quad \frac{1}{2}(1+i, \pm j \pm k) .
$$

A local section which excludes only the fibre $\infty$ is

$$
(0,1), \quad \frac{1}{2}( \pm 1 \pm i, 1+i), \quad \frac{1}{2}( \pm j \pm k, 1+i) .
$$

Right multiplication of $\frac{1}{2}(1+i, q)$ by $q^{-1}(1+i)$ gives $\frac{1}{2}\left((1+i) q^{-1}(1+i), 1+i\right)$, and effects the transition between the sections (4.6) and (4.7) where they overlap. $\frac{1}{2}(1+i, q)$ is on the fibre $Q=(1+i) q^{-1}$, and $q^{-1}(1+i)=(1+i)^{-1} Q(1+i)$, so the transition function between the sections is $Q \rightarrow(1+i)^{-1} Q(1+i)$. This is not the identity map but it is a $1-1$ map from the set $\{ \pm 1, \pm i, \pm j, \pm k\}$ to itself which may be regarded as a discrete map of degree 1, analogous to the map of degree 1 that occurs in the smooth $S U(2)$ Hopf bundle.

The holonomies on triangles may be studied in the same way as in the discrete $U(1)$ Hopf bundle. They are not as invariant as before. The holonomy is conjugated by an element of the gauge group when the starting point in the bundle is changed. We have, unfortunately, not been able to identify the discrete analogue of the second Chern form. It may be possible to define it in terms of the holonomies on the triangular faces of the 32 elementary 4-dimensional simplexes which make up the base space, or possibly in terms of holonomies on more complicated loops. The second Chern number, which one expects to be 1 for all connections, would be obtained by summing over these simplexes. Presumably, as in the smooth $S U(2)$ Hopf bundle, it would be related to the map between overlapping local sections described above.

Acknowledgement. I would like to thank Ron Horgan for encouraging me to look once more at the problems of lattice gauge theory.

\section{References}

1. Lüscher, M.: Topology of lattice gauge fields. Commun. Math. Phys. 85, 39 (1982)

2. Woit, P.: Topological charge in lattice gauge theory. Phys. Rev. Lett. 51, 638 (1983)

3. Phillips, A., Stone, D.: Lattice gauge fields, principal bundles and the calculation of topological charge. Commun. Math. Phys. 103, 599 (1986)

4. Teper, M.: Topological susceptibility in $S U(2)$ lattice gauge theory: an exploratory study. Phys. Lett. 171 B, 86 (1986)

5. Eguchi, T., Gilkey, P.B., Hanson, A.J.: Gravitation, gauge theories and differential geometry. Phys. Rep. 66, 213 (1980)

6. Steenrod, N.: The topology of fiber bundles. Princeton, NJ: Princeton Univ. Press 1951

7. Coxeter, H.S.M.: Regular polytopes. London: Methuen 1948

8. Wilson, K.G.: Confinement of quarks. Phys. Rev. D 10, 2445 (1974)

9. Gosset, T.: On the regular and semi-regular figures in space of $n$-dimensions. Mess. Math. 29, $43(1900)$

10. Hurwitz, A.: Über die Zahlentheorie der Quaternionen. Math. Werke 2, 303 (1933)

Communicated by L. Alvarez-Gaumé 
